\title{
Characteristics of 488 Megacycles Per Second Radio Signals Reflected From the Moon
}

\author{
B. C. Blevis and J. H. Chapman
}

(January 18, 1960)

\begin{abstract}
Radio signals at 488 megacycles per second, received after reflection from the moon, have been studied using a continuous wave bistatic radar system located near Ottawa, Canada. These experiments were carried out during 1957 and consisted of monitoring the signals received on two orthogonal dipoles mounted at the focus of a 28 foot parabolic tracking antenna. The total mean received signal yielded an effective radar cross section of the moon at this frequency of 0.05 of the projected area. The libration fading as observed in the two orthogonal receiver channels was in synchronism, with a cross-correlation coefficient of 0.89 . It was established that the signal is not depolarized significantly on reflection at the surface of the moon or in passing through the ionosphere. Rotation of the plane of polarization of the radio wave in the double passage through the ionosphere was observed to change by nearly 180 degrees over a six-hour period during quiet ionospheric conditions.
\end{abstract}

\section{Introduction}

Signals reflected from the moon have been used for studying propagation conditions along the path between the earth and the moon and for studying the radio reflection characteristics of the moon itself $[1,2,4] .^{2}$ The study reported in this paper was begun primarily to investigate the rotation of the plane of polarization and the attenuation of UHF signals traversing the ionosphere. For this reason, plane polarized waves were transmitted from a tracking antenna, and the signals which were received after reflection from the moon were monitored by two independent receiving systems fed from identical orthogonal dipoles mounted in a second tracking antenna. By this means, the rotation of the plane of polarization could be observed and measured over a period of time.

The measurements were begun early in 1957 and were continued throughout the year, with various changes in equipment and procedure. Both longpulse [2] and continuous-wave transmissions were used. For the latter measurements, it was necessary to separate the transmitter and receivers to prevent the very strong transmitted signal from saturating the receivers. For the $\mathrm{cw}$ measurements described in this paper, the separation was approximately 60 miles. Provided the direct signal was sufficiently low, the moon-reflected signal could be distinguished by means of its Doppler shift.

\section{Experimental Method}

The equipment used during these experiments is described briefly in the following paragraphs.

The transmitter was operated at a frequency of $488 \mathrm{Mc}$, with a cw power output of $10 \mathrm{kw}$. The

Contribution from Defence Research Telecommunications Establishment, Shirley Bay, Ottawa, Canada.

2 Figures in brackets indicate the literature references at the end of this paper. transmitting and receiving antennas used 28-ft parabolic reflectors with equatorial mounts and drive systems. Small correction motors on each axis permitted uninterrupted tracking of the moon from moonrise to moonset. The transmitted radiation was essentially plane-polarized.

Two identical receiving systems with noise figures of about $7 \mathrm{db}$ were fed from orthogonal dipole antennas mounted at the focus of the receiving parabolic reflector.

The transmitter frequency and the first two local oscillator injection frequencies of the receiver were controlled from similar crystal standards with an overall stability of the order of 1 part in $10^{8}$. The receiver output at the second $\mathrm{IF}^{\mathrm{w}}$ was then mixed with the signal from a stable $\mathrm{VFO}$ to give a third IF at a center frequency of $425 \mathrm{cps}$. The varying Doppler shift [2] due to the relative motion of the moon and the point of observation was eliminated by continual adjustment of the VFO to maintain a stable Lissajous pattern on an oscilloscope. In this way, the 425-cps output could be passed through a fixed narrow band $(90 \mathrm{cps})$ filter. The signal was then recorded on magnetic tape for subsequent analysis, and also detected and displayed on paper chart recordings.

\section{Signal Characteristics}

\subsection{Faraday Rotation}

The Faraday rotation of the plane of polarization of a radio wave passing through the ionosphere has been described in detail in the literature [1]. A recording which exhibits the effects of Faraday rotation is given in figure 1 . This record was made during a period of relatively quiet ionospheric conditions. The slow rotation of the plane of polarization of the signal during a 6 -hr period is evident from a comparison of the two traces which come 


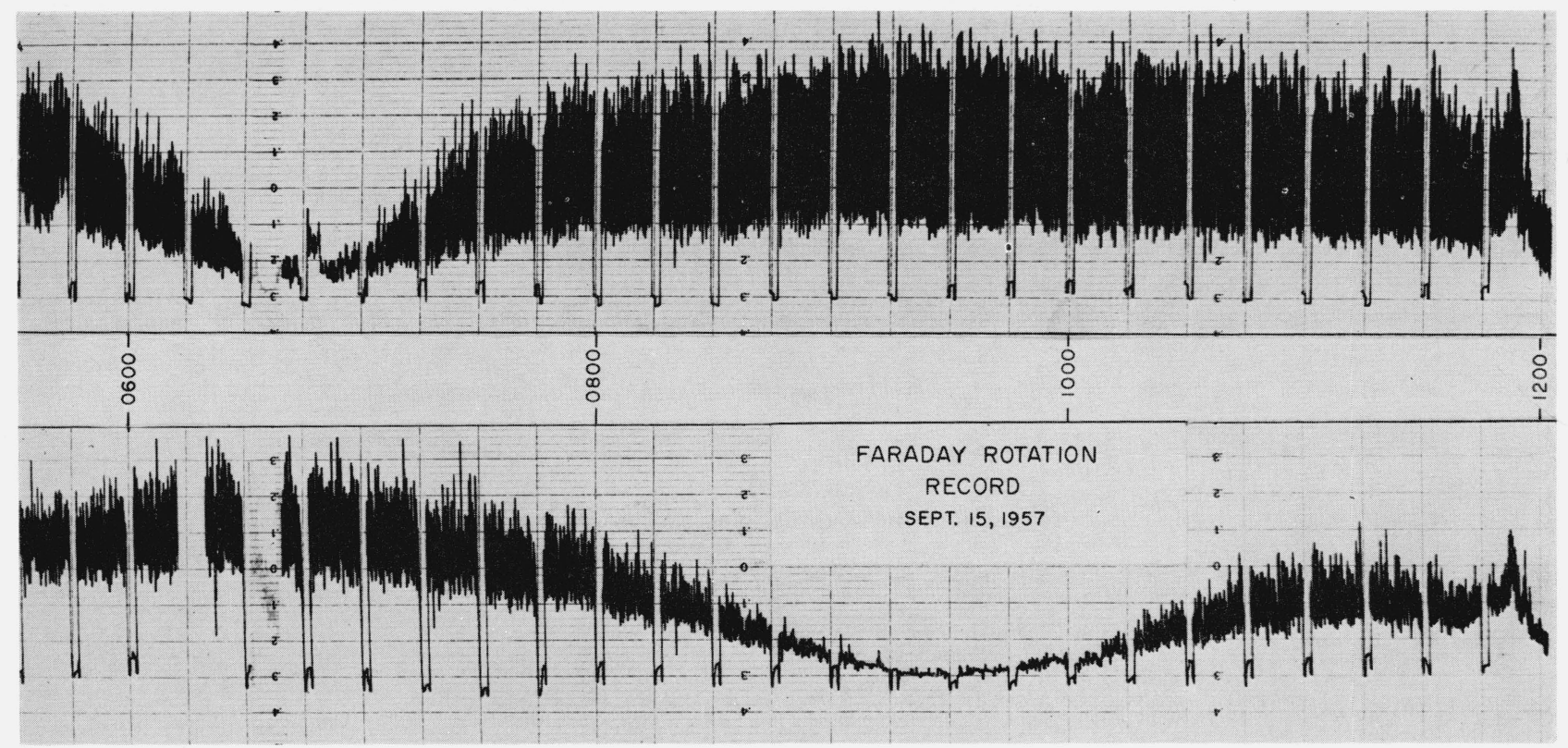

FiguRe 1. Faraday rotation record.

Comparison of the relative signal on two orthogonal polarizations over a 6-hr period on 15 September 1957.

from the two orthogonal receiver channels. The broadening of the record is due to the libration fading, as discussed below, which is partially smoothed by post-detection integration. Timing marks on the record were made by shutting the transmitter off every $15 \mathrm{~min}$. Receiver noise can be seen during these periods.

Upper transit of the moon occurred just before the beginning of this record, and the moon was tracked down to the horizon. The increase of signal near the end is attributed to constructive interference between the direct and ground-reflected signals just before moonset.

This record was obtained during the morning when the total electron content of the ionosphere was increasing towards its maximum. In addition, the amount of rotation due to the increasing path length through the ionosphere was also increasing. It is reasonable to assume, therefore, that the rotation of the plane of polarization was monotonic, and hence a total change in rotation of almost $180 \mathrm{deg}$ is indicated.
The difference in signal levels between the two orthogonal channels during periods when the received signal was nearly all in one of the two channels, or between maximums and minimums on a single channel, has been observed to be as great as $16 \mathrm{db}$. The isolation between the feed dipoles was $40 \mathrm{db}$ when measured alone, and although this value was somewhat reduced when the dipoles were mounted in front of the reflector, the isolation was still greater than the measured differences between channels.

\subsection{Libration Fading}

Fading due to libration of the moon is generally deep and rapid, being as fast as several cycles per second during periods of maximum libration rate. This fading is due, of course, to the change in relative path lengths of signals scattered from different portions of the moon's surface.

A typical expanded recording covering a 35-sec period is shown in figure 2 . The signal was about

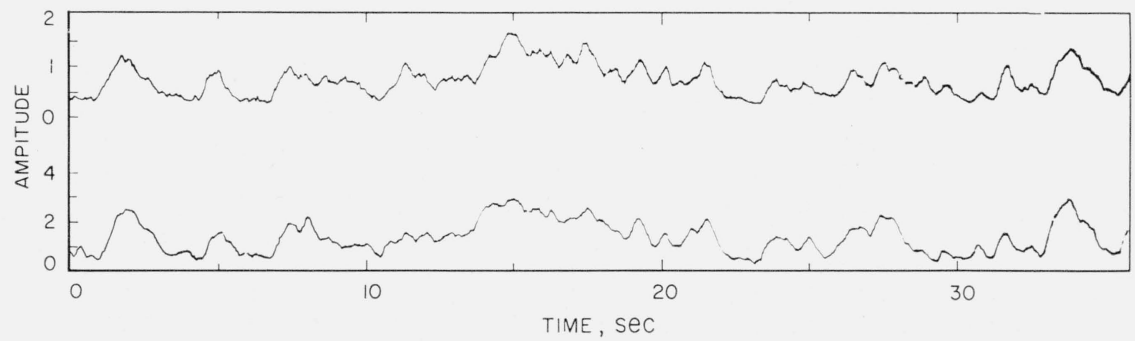

Figure 2. Libration fading.

A 35-sec recording of the amplitude of signals on two orthogonal polarizations. The recording time constant was 0.4 sec. 
wice as strong in the upper channel but an adustment of the recorder gain has compensated for his. The depth of fading will be limited by the effective bandwidth or amount of post-detection ntegration. However, in this example, with a ime constant of $0.4 \mathrm{sec}$, the signal still fades into ooise on occasion.

The signals are seen to be well correlated in the two receiving channels. Several analyses of this correlation have been performed. A typical example s illustrated by figure 3 which shows a scatter plot of the signal amplitude on the two channels at 0.8ec intervals during a 1-min period. Machine calculations indicate a cross-correlation coefficient of 0.89.

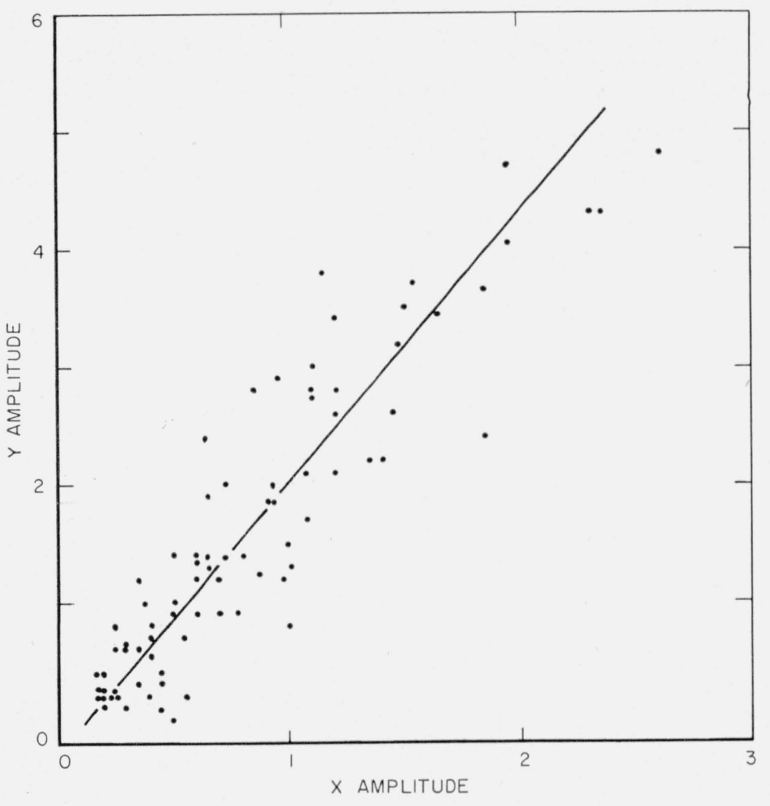

FIGURE 3. Scatter plot of the relative signal amplitude on orthogonal polarizations.

An amplitude of zero corresponds to the noise level.

The amplitude distribution of the signal on either channel approximates that of a Rayleigh distribution as can be seen by figure 4 , which is a ogarithmic plot of the probability density in amplitude. The amplitude is given in arbitrary units. These data were obtained by sampling the amplitude of a 5 -min section of record at a sampling frequency of $1 \mathrm{kc}$.

From the analyses in this and the above section, one can conclude that there is little depolarization of he signal on reflection at the moon's surface or in ts passage through the ionosphere. The plane polarized signal, which was transmitted to the moon, s received on the earth with the plane polarization argely preserved.

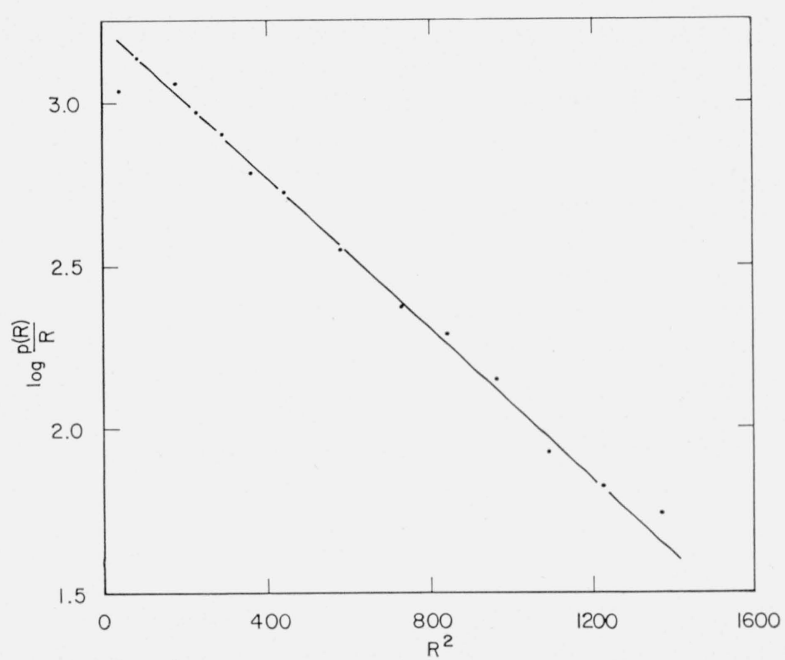

Figure 4. Log plot of the probability density function. $R$ is the signal amplitude on a single channel in arbitrary units, $p(R)$ its probability density.

\subsection{Total Signal Power}

A number of calculations have been made of the total signal power received by adding vectorially the signals in each channel. Assuming antenna gains of $30 \mathrm{db}$, the corresponding effective cross section of the moon is calculated to be of the order of $5 \times 10^{11} \mathrm{~m}^{2}$ or $0.05 \pi a^{2}$, where $a$ is the lunar radius. The uncertainty in this result is believed to be less than $3 \mathrm{db}$.

When these experiments began in February 1957, there were times when the total signal was unaccountably low for periods of the order of an hour or so. The apparent loss of signal was about $6 \mathrm{db}$ or more. The times when this phenomenon occurred did not appear to correlate with the position of the moon above the horizon [3]. Investigations at the time were thought to rule out the possibility of any equipment malfunction.

Subsequently fairly exhaustive studies have been made in an attempt to repeat these observations under carefully controlled conditions using superior equipment [4]. The results have been negative, in that the total power was observed to remain sensibly constant within a decibel or so. It is concluded that either the physical conditions responsible for the earlier loss of signal were not duplicated during the subsequent tests, or that some still unknown equipment factor was responsible.

\section{Conclusions}

The Faraday rotation of the radio signal traversing the ionosphere in two directions has been observed to change by nearly 180 deg over a 6 -hr period during quiet ionospheric conditions.

From an analysis of the rapid fading of the signal due to libration of the moon and of the difference in signal power between cross-polarized components, 
it is concluded that the transmitted polarization is largely preserved on reflection from the moon's surface and that the surface is probably not deeply serrated and rough, at least at this wavelength (62 $\mathrm{cm}$ ).

The effective value of the lunar radar cross section at $488 \mathrm{Mc}$, obtained by adding vectorially the mean signal on orthogonal polarizations, is of the order of $0.05 \pi a^{2}$ as measured with a $\mathrm{cw}$ radar.

The results of more recent experiments carried out jointly by Lincoln Laboratory of M.I.T., the Stanford Research Institute, and the Defence Research Telecommunications Establishment have been published elsewhere [4].

The authors acknowledge the assistance of their colleagues, particularly F. D. Green, H. V. Serson, and E. A. Cameron, in making these measurements.

\section{References}

[1] I. C. Browne, J. V. Evans, J. K. Hargreaves, and W. A. Murray, Radio echoes from the moon, Proc. Phys Soc. B 69, 901 (1956).

[2] B. C. Blevis, Ionospheric studies by the lunar rada technique, Nature 180, 138 (1957).

[3] S. Rush and L. Colin, The effects on radio astronomica observations due to longitudinal propagation in th presence of field-aligned ionization, Proc. I.R.E. 46 356 (1958).

[4] J. C. James, L. E. Bird, R. P. Ingalls, M. L. Stone J. W. B. Day, G. E. K. Lockwood, and R. I. Presnell Observed characteristics of an ultra high frequenc signal traversing an auroral disturbance, Nature $\mathbf{1 8 5}$ $510(1960)$. 